\title{
A three-parameter model for fatigue crack growth data analysis
}

\author{
A. De Iorio, M. Grasso, F. Penta, G.P. Pucillo \\ Università di Napoli Federico II, Dipartimento di Meccanica ed Energetica,Via Claudio 21 - 80125 Napoli \\ antdeion@unina.it
}

\begin{abstract}
A three-parameters model for the interpolation of fatigue crack propagation data is proposed. It has been validated by a Literature data set obtained by testing $180 \mathrm{M}(\mathrm{T})$ specimens under three different loading levels. In details, it is highlighted that the results of the analysis carried out by means of the proposed model are more smooth and clear than those obtainable using other methods or models. Also, the parameters of the model have been computed and some peculiarities have been picked out.
\end{abstract}

KEYWORDS. Fatigue crack growth; Data analysis; Non-linear regression.

\section{INTRODUCTION}

$\mathrm{T}$ he assessment of the fatigue damage by means of phenomenological models notoriously is closely linked to the analysis of experimental results obtained from standard specimens of a given material tested with suitably chosen loading programs. However, it is well known that crack growth data have stochastic nature, as the phenomenon which generates them, due to, essentially, the random evolution of the local combinations, at each point of the crack front, of the induced stress state and material properties. For this reason, in order to identify a deterministic law describing the phenomenon, that is able to represent the common data trend independently of the global scatter or the position of the single data point, data have to be elaborated by means of a best-fit method after having selected an analytical model. Since the availability of a reliable crack propagation model has a tremendous impact on the fatigue design practice, for over half a century this problem has been faced by many researchers [1]. For the same reason, the attention of the researchers has been focused also on the accuracy by which the experimental data are produced and analysed, with the aim of defining a standard procedure for material testing and related results analysis (ASTM) and to formulate interpretative models of the experimental data.

The most used methods for crack growth testing and data analysis are those suggested by ASTM Standard [2], which, concerning the data analysis, proposes two different approaches: the Secant Method and the Incremental Polynomial Method. Since both methods are based on local interpolation of experimental data, irregularities and/or anomalies in the data distribution, for the first method, and the number of data points chosen for the best-fit parabola, for the second one, affect significantly the results.

On the other hand, the foremost polynomial or exponential analytical formulas found in Literature [3-6] do not seem to have solved completely the problem. Hence our interest in facing this problem, in order to contribute, if possible, to improve on the quality of raw crack propagation data analysis by formulating an interpretative model for the whole lives field of the acquired data.

\section{FORMULATION OF THE MODEL}

mong the various analysis methods developed till now, besides those proposed by the ASTM Standards, we mention for their popularity:

- Mukherjee Method [3], which is based on the linear interpolation of the finite differences computed between 
two consecutive points, in order to estimate the rate of growth at the central point;

- polynomial expressions of Smith [4], Davies and Feddersen [5], that interpolate locally or globally the experimental data;

- Polak and Knesl Method [6], which uses splines to fit and reduce the scatter in the data.

Most of the methods until now proposed are local interpolations of experimental points, as already stated in the introduction, and are strongly affected by the real distribution of the analysed data, being unable to filter both the irregularities and anomalies that are always present in the sample. Therefore, most analytical models derived in such a way are very often closely linked to the particular experimental practice adopted to produce the data and, above all, do not fit all data with the needed and controlled accuracy in the whole cycles number range of each test. In a previous paper [7], some of the Authors already discussed the possibility to use splines of an arbitrary order to globally interpolate the raw data and reduce the scatter of the crack growth material constants when gapped or noisily data are used.

In order to formulate a new model able to (at least) partially fill the aforementioned lacks, the results of $180 \mathrm{crack}$ growth tests carried out by Ghonem and Dore [8] have been analysed. To find the curve that better interpolates all the examined data, different analytical formulations have been tested. By means of successive refinements, the following three parameters model has been defined:

$$
a=\frac{a_{0}+\gamma}{\left(1-\frac{(\alpha \cdot N)}{N_{f}}\right)^{\beta}}
$$

where:

$a_{0}=$ initial crack length;

$N_{f}=$ number of cycles to failure;

$\alpha, \beta, \gamma=$ model parameters identified by a non-linear least squares method;

$N=$ number of cycles corresponding to a given crack length.

The crack growth rate can be directly evaluated in the range $\left[\mathrm{N}_{0}-\mathrm{N}_{\mathrm{f}}\right]$ by means of the previous expression without amplify the irregularities and/or anomalies of the raw data and without losing information in the initial and final part of the aforementioned range, as it occurs with other procedures as those suggested by ASTM Standards.

\section{VERIFICATION OF THE MODEL}

he data of the 180 tests of Ghonem and Dore, which are fully described in term of both specimens and testing conditions in the paper [8], are summarized in Fig. 1-3. Each figure reports the results of a series of 60 tests carried out under the same loading conditions.

Once the model has been defined, the goodness-of-fit has been evaluated computing the residuals and the values attained by the coefficient of determination $\mathrm{R}^{2}$, which is reported in the graphs of Fig. 4-6, for each data set.

For a quick (non-objective) evaluation of the goodness of the interpolation, the results of some tests together with the related best-fitting curves obtained by the proposed model are also reported in Fig. 7 .

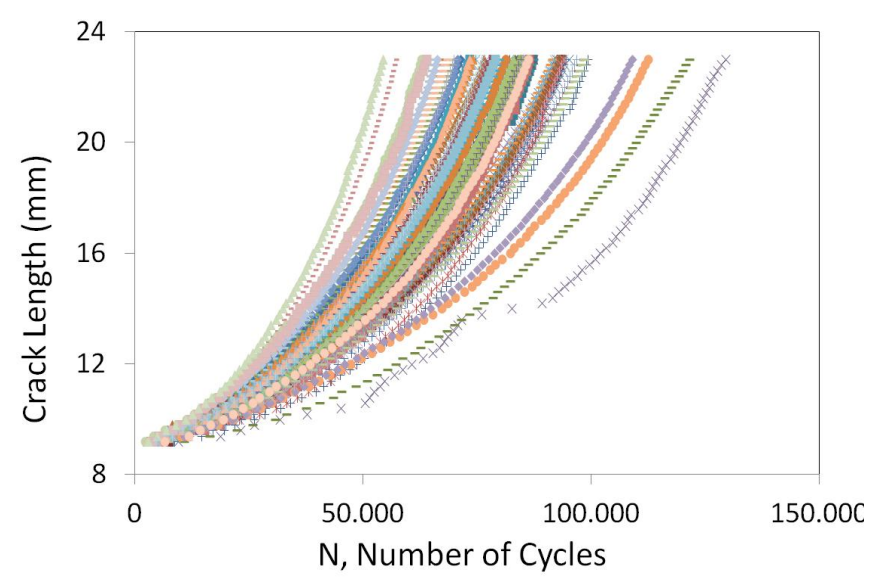

Figure 1: Fatigue crack growth curves set I $(\mathrm{R}=0.6)$.

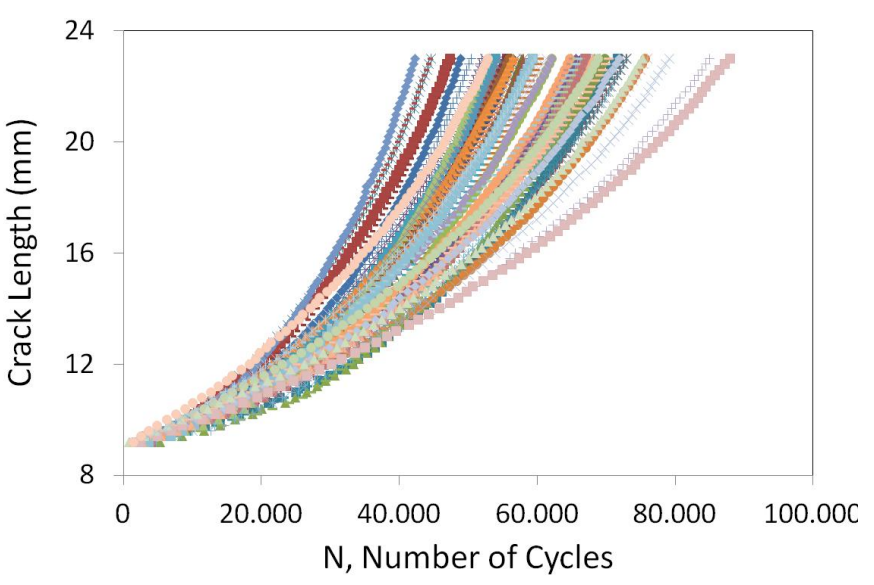

Figure 2: Fatigue crack growth curves set II $(\mathrm{R}=0.5)$. 


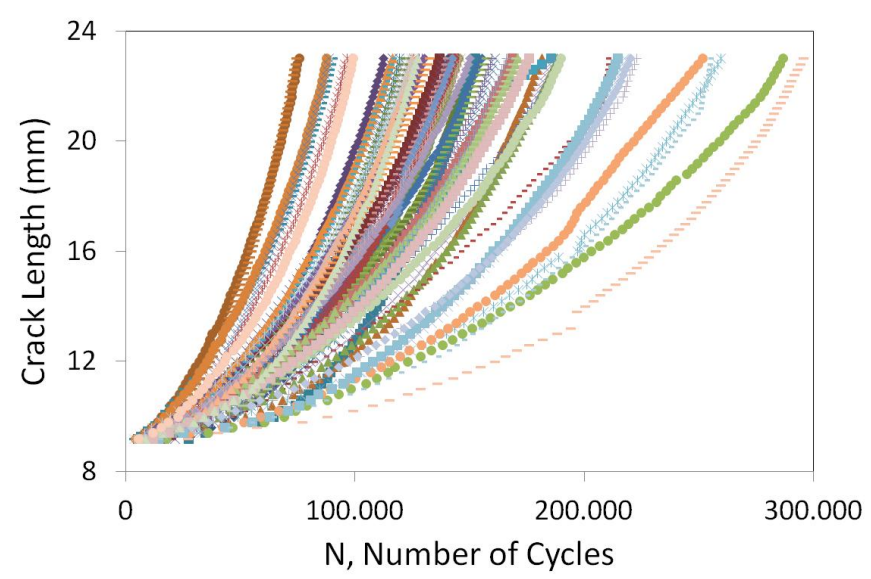

Figure 3: Fatigue crack growth curves set III $(\mathrm{R}=0.4)$.
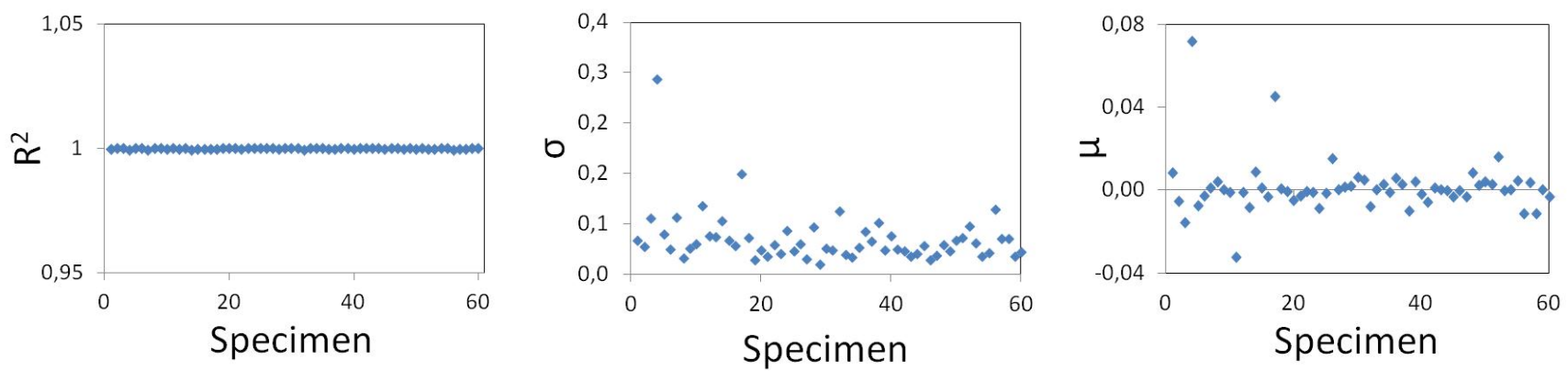

Figure 4: Coefficient of determination, mean and standard deviation for set I.
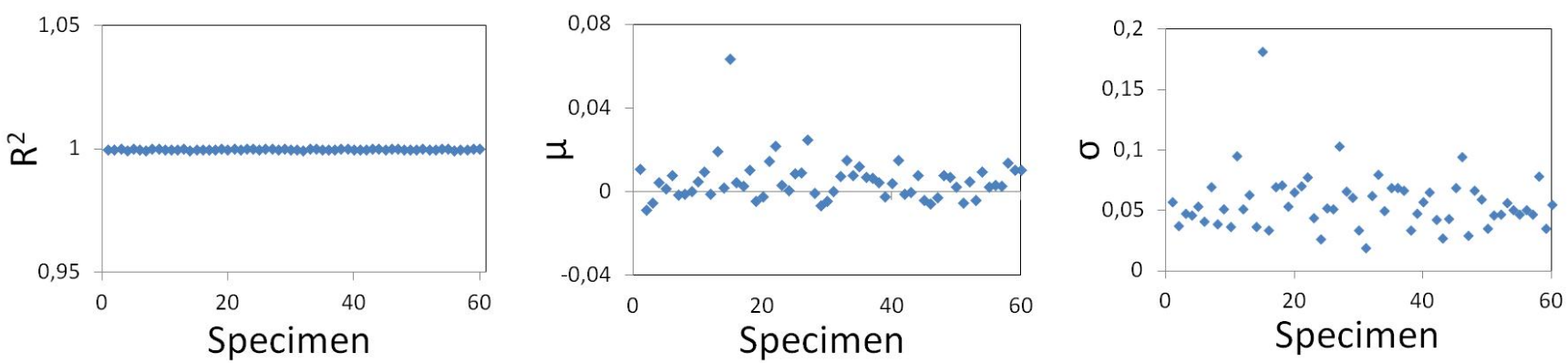

Figure 5: Coefficient of determination, mean and standard deviation for set II.
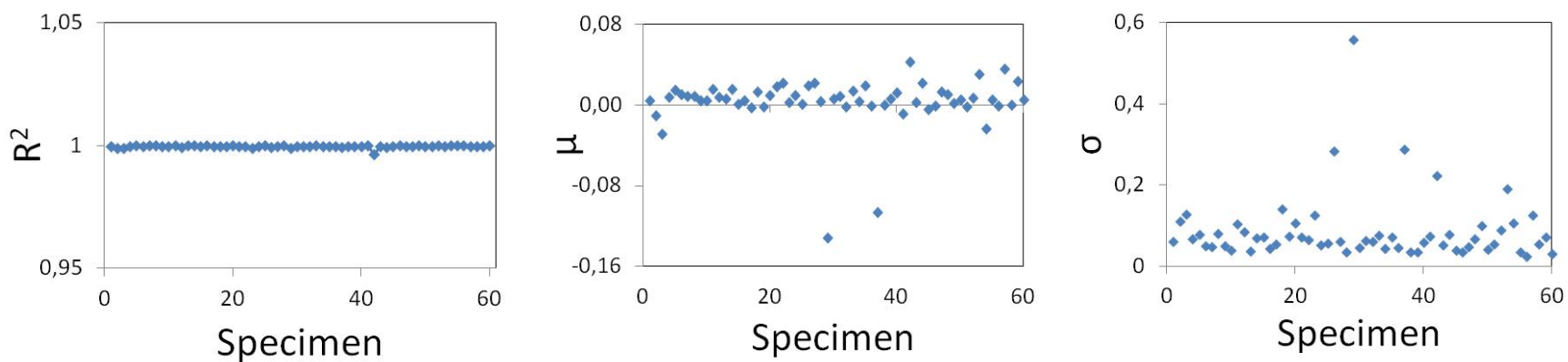

Figure 6: Coefficient of determination, mean and standard deviation for set III. 


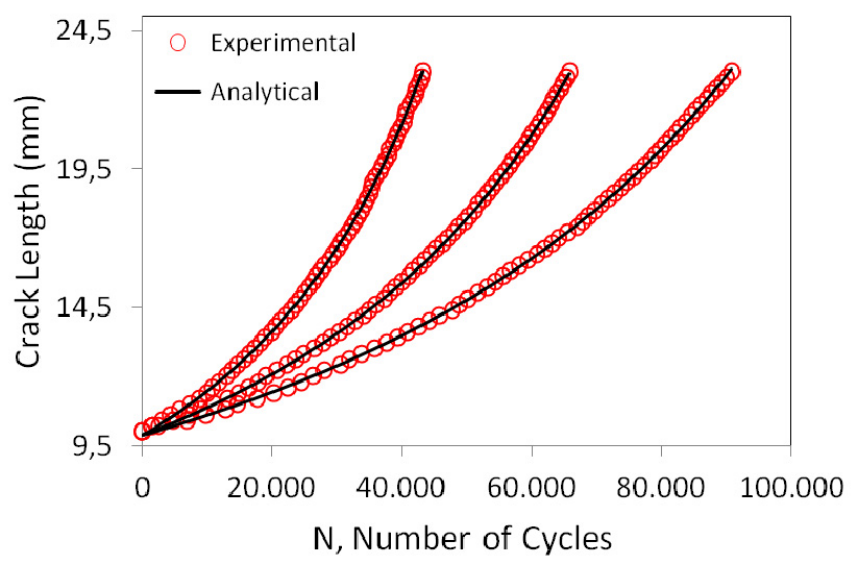

Figure 7: Crack growth data with best-fit curves.

Moreover, normality tests of the residuals have been carried out using the $\chi^{2}$ test and the frequency distributions of the residuals computed for each specimen tested have been also diagrammed to verify that the mean was close to zero and the standard deviation was very small, in other words that the conditions to guarantee a small error in the estimation of the crack lengths by means of the model were satisfied [9]. In Fig. 8, as an example, three of the aforementioned distributions are reported, one for each set.
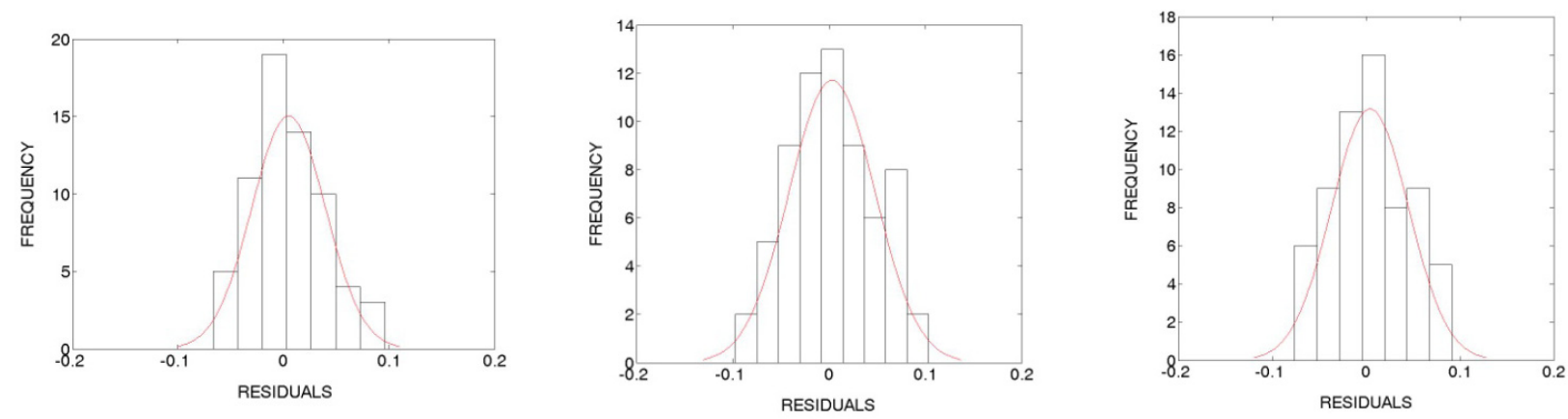

Figure 8: PDF of residuals of three selected best-fit, one for each set.

From the examination of the normality test results, it has been noted that the majority of them verify the expected conditions (see. Tab. 1), whereas the others do not pass the test due to some large anomalies present in the frequency distribution diagrams. However, if the graphs of the raw data are observed in details in the fields corresponding to each anomaly, it can be seen that the data points move away from the trend of all other points in either an irregular or anomalous manner.

\begin{tabular}{lcc}
\hline & $\begin{array}{c}\text { Numbers of best-fit with } \\
\text { normal } \\
\text { distribution of residuals }\end{array}$ & $\begin{array}{c}\text { Numbers of best-fit with } \\
\text { non normal } \\
\text { distribution of residuals }\end{array}$ \\
Set_I & 47 & 13 \\
Set__ II & 32 & 28 \\
Set_III & 31 & 29 \\
\hline
\end{tabular}

Table 1: Results of normality tests.

The outliers in the graphs of the mean and standard deviation are not only due to the random scatter in the raw data, but also to the fact that in some experimental curves there are data points sequences that do not follow the trend of the whole test. This anomaly affects the response of the optimization algorithm used in the non-linear least square method. The best-fitting model parameters obtained for these latter experimental curves define a solution with a residual distribution 
distorted in mean and asymmetric.

To highlight what said, three curves with irregular points and a curve with anomalous data, in which the residuals distribution exhibits an unbalanced mean and a marked asymmetry due to the solely effect of the irregular data in the anomalous part (spotlighted), are reported in Fig. 9-11 and Fig. 12, respectively.

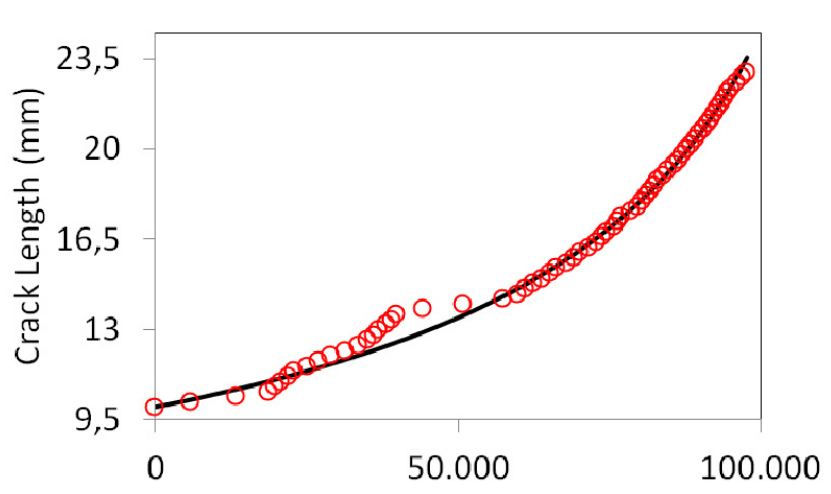

$\mathrm{N}$, Number of Cycles

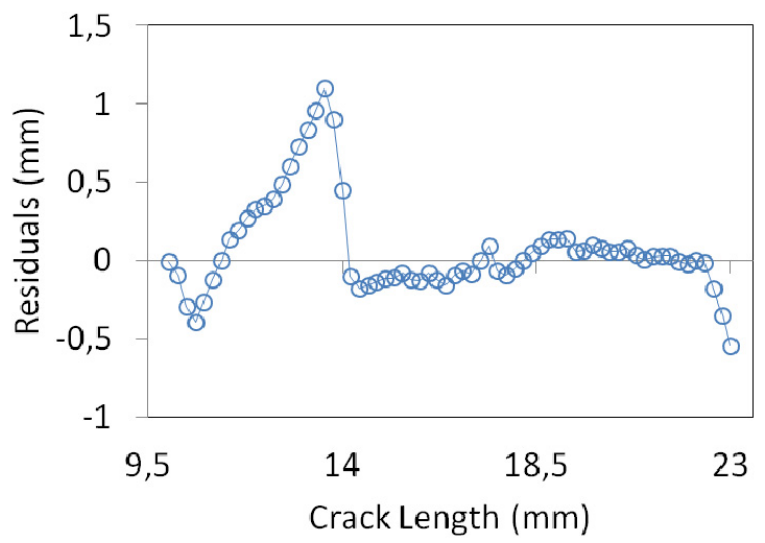

Figure 9: Crack length vs. Number of Cycles (left) and corresponding residuals (right).
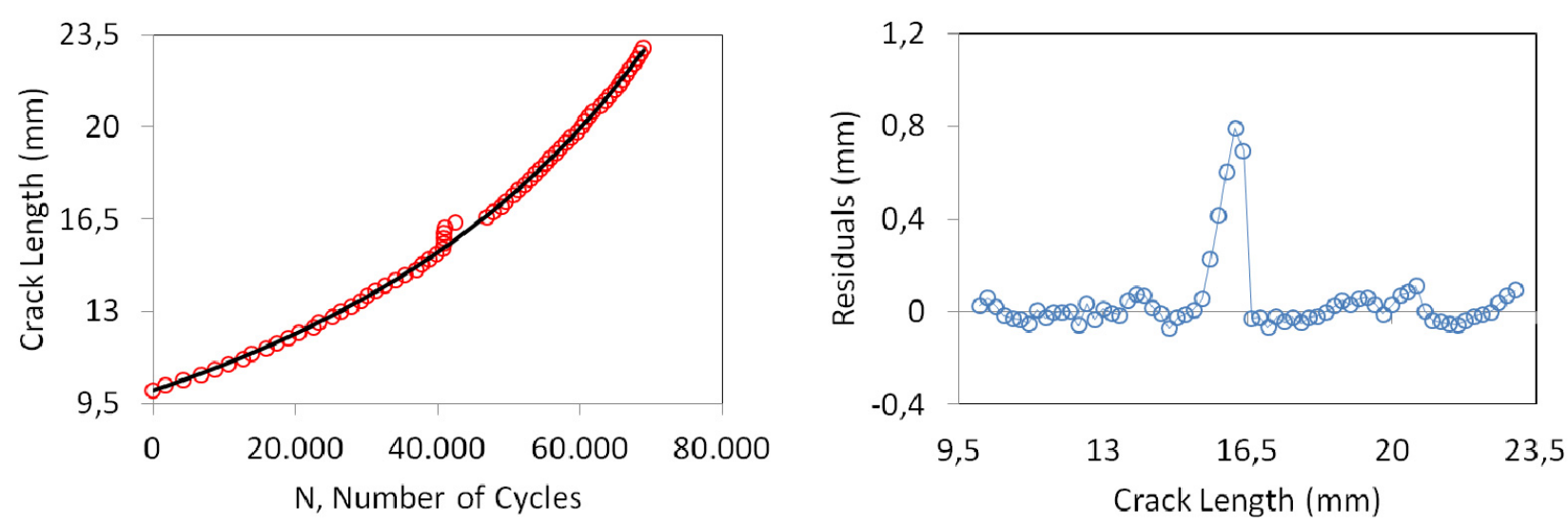

Figure 10: Crack length vs. Number of Cycles (left) and corresponding residuals (right).
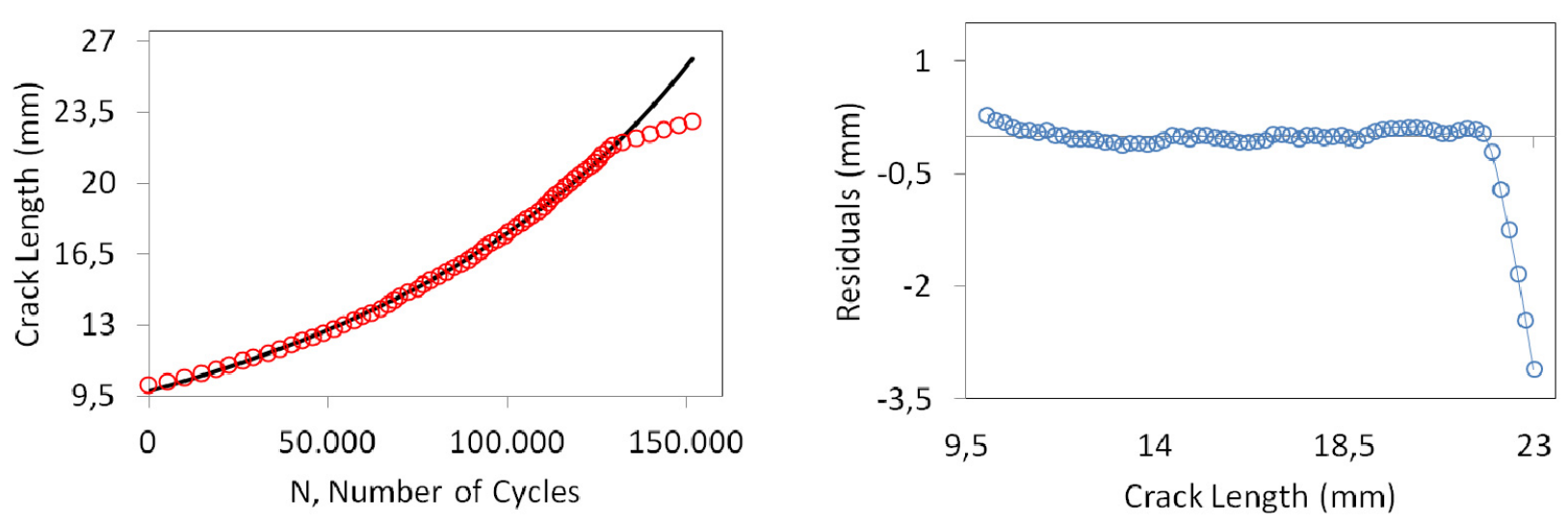

Figure 11: Crack length vs. Number of Cycles (left) and corresponding residuals (right). 

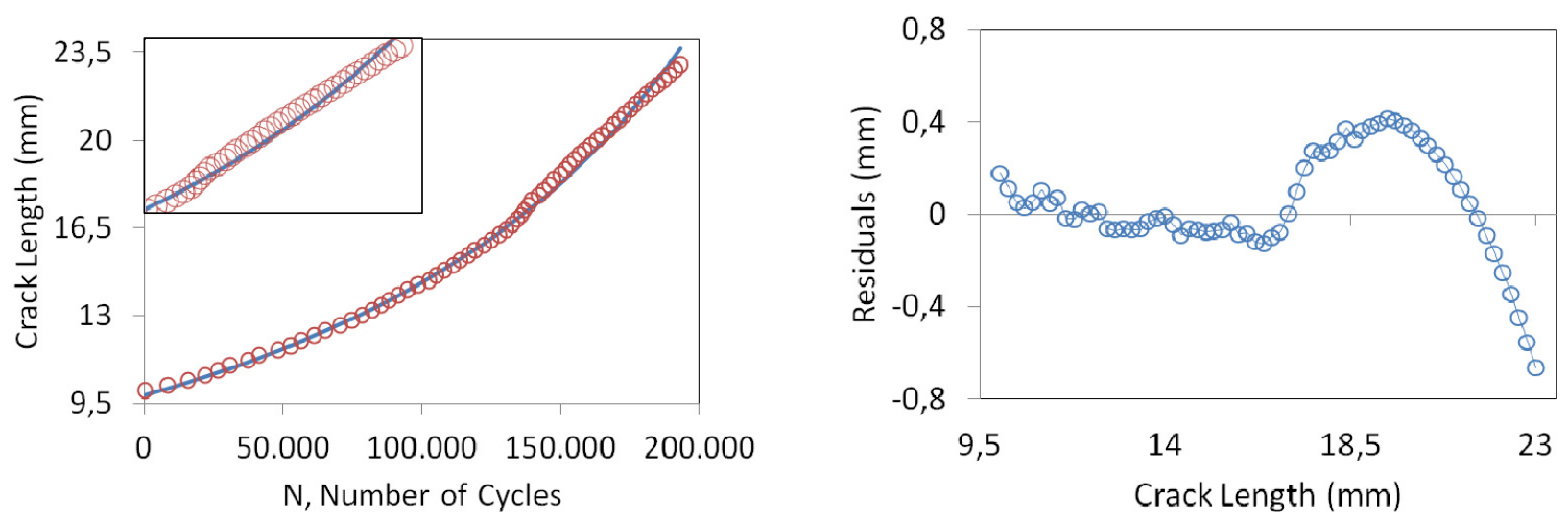

Figure 12: Crack length vs. Number of Cycles (left) and corresponding residuals (right).

In any case, the best-fitting with the proposed method appears visually acceptable and the coefficient of determination $\mathrm{R}^{2}$ is very close to one. It follows that if the normality test of the residuals distribution does not verify the optimum conditions for the approximation of the experimental data with the proposed model, it is due to the defectiveness of some experimental points or of short parts of some experimental curves. This result, apparently negative, can even be profitably used to filter the experimental data, that is eliminate all data that move away in an excessive or anomalous manner from the unique natural trend of all the other data points, as unique must be the physical law that characterizes the crack propagation phenomenon in each material that is free of geometrical singularities and structural inhomogeneities.

This way to see the experimental data allows minimizing and improving the information derivable from testing and, in this particular case, to improve the evaluation of the model parameters $\alpha, \beta, \gamma$ using all available data.

\section{IDENTIFICATION AND VARIABILITY OF THE PARAMETERS $\alpha, \beta, \gamma$}

$\checkmark$ he interpolation of the experimental data of each test, through the proposed model and best-fitting technique, allows identifying the parameters $\alpha, \beta, \gamma$ of the model. The numerical values of these parameters are represented in three distinct groups of three diagrams, each group being relative to a data set obtained under the same loading condition (Fig. 13-15). The parameters values assigned to each data set are the statistical averages of the values obtained for each experimental curve of the set.

They are given in Tab. 2, where the standard deviations of the three samples of parameter estimates are also reported.

\begin{tabular}{ccccc}
\hline & & $\boldsymbol{\beta}$ & $\boldsymbol{\alpha}$ & $\boldsymbol{\gamma}$ \\
\hline Set_I & $\mu$ & 1.37 & 0.47 & -0.062 \\
& $\sigma$ & 0.35 & 0.078 & 0.073 \\
\hline Set_II & $\mu$ & 1.55 & 0.43 & -0.105 \\
& $\sigma$ & 0.42 & 0.069 & 0.15 \\
\hline Set_III & $\mu$ & 1.62 & 0.43 & -0.15 \\
& $\sigma$ & 0.53 & 0.01 & 0.176 \\
\hline
\end{tabular}

Table 2: Model parameters values. 

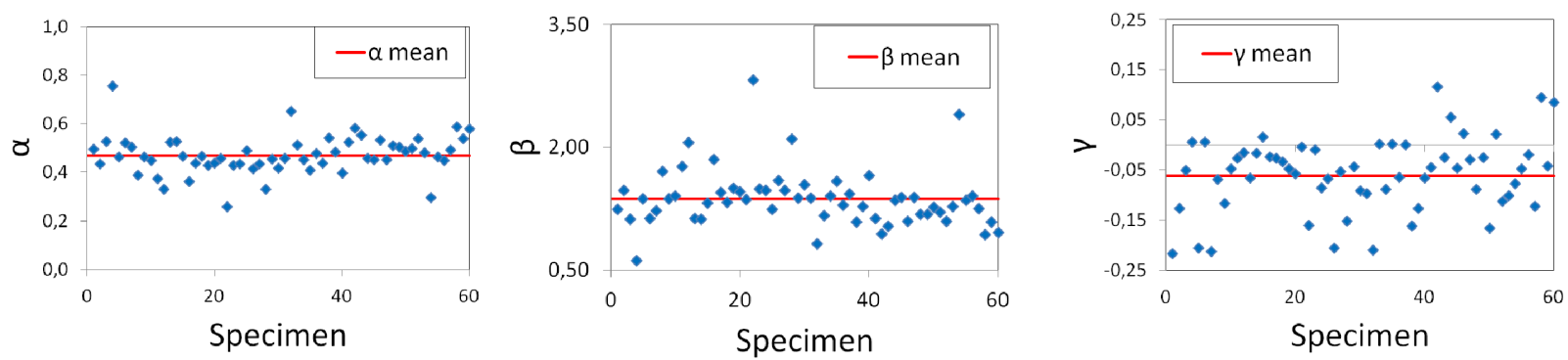

Figure 13: $\alpha, \beta, \gamma$ parameters with corresponding mean value for set $\mathrm{I}$.
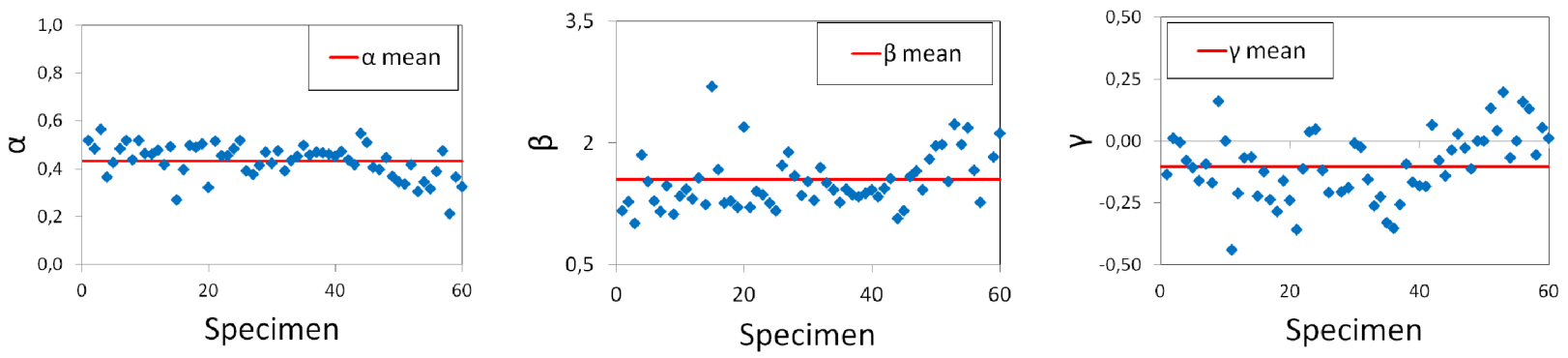

Figure 14: $\alpha, \beta, \gamma$ parameters with corresponding mean value for set II.
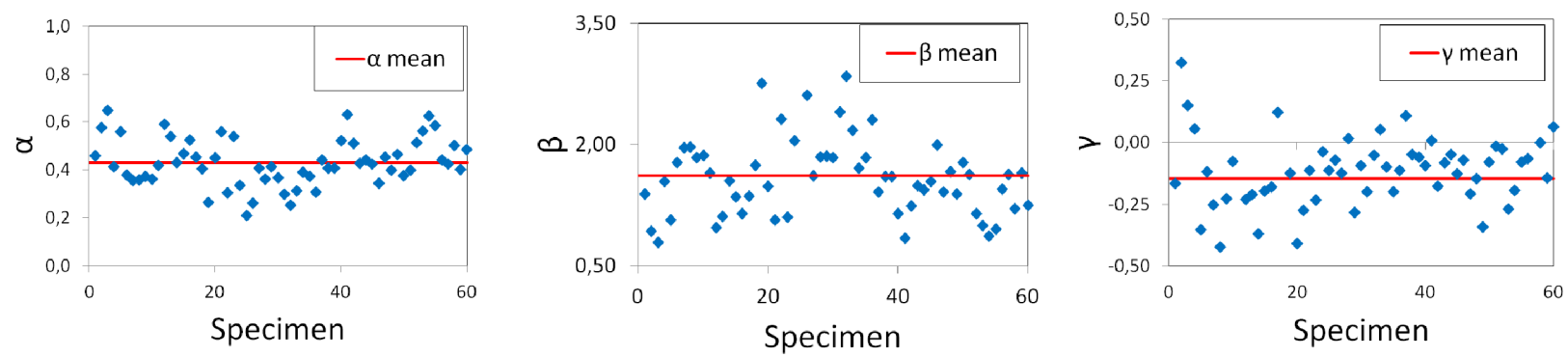

Figure 15: $\alpha, \beta, \gamma$ parameters with corresponding mean value for set III.

From the achieved results it can be seen that the parameter $\alpha$ has a small deviation around the mean value in respect to that of $\beta$ and $\gamma$. Also, the two parameters $\beta$ and $\alpha$, plotted one as function of another (see Fig. 16), are related with the following law:

$$
\beta=\frac{C}{\log (1-\alpha)}
$$

In Fig. 16, for each data set, the values of the constant $C$ together with the fitting curves are reported; in order to show the goodness of fit, also the values of the coefficient of determination $\mathrm{R}^{2}$ are reported in the same figure.

Moreover, since the constant $\mathrm{C}$ remain almost unchanged for the three data sets, it could be considered as a material constant, appearing independent from the loading conditions that differentiate the three testing groups.
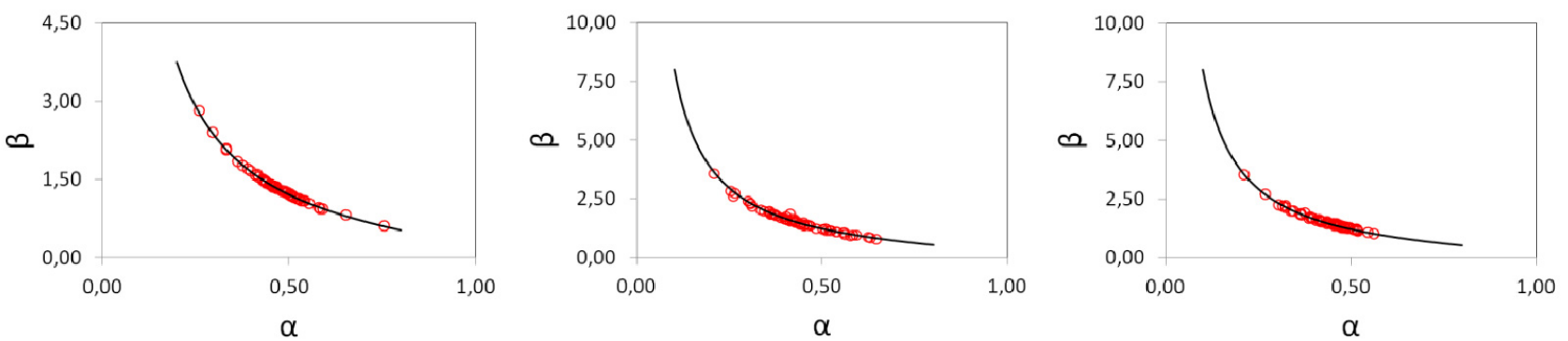

Figure 16: Correlation between $\alpha$ and $\beta$ values for each set. 
Further relations seem to be detectable between the loading ratio $\mathrm{R}$ and the parameters $\beta$ and $\gamma$, on the one end, and between the parameter $\gamma$ and the constant $C$, on the other end, as it can be seen in Fig. 17 and 18.

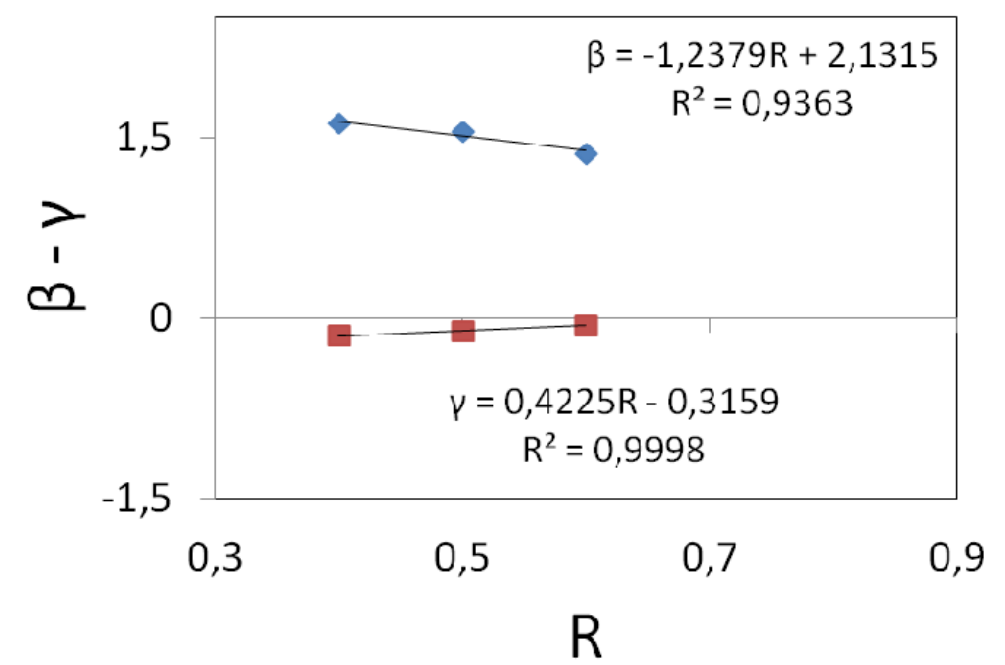

Figure 17: Correlation between $\beta, \gamma$ values with the loading ratio.

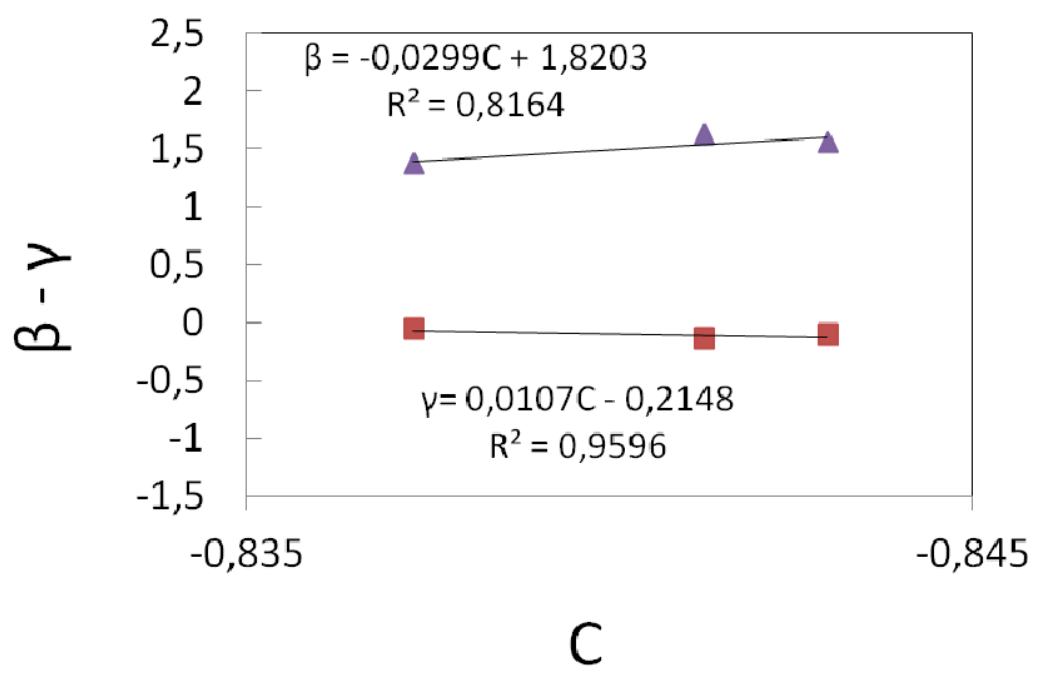

Figure 18: Correlation between $\beta, \gamma$ values with the constant $C$.

Obviously, about these latter relations, further experimental investigations on other materials and with a greater number of loading conditions are needed.

\section{CONCLUSIONS}

A $\mathrm{n}$ analytical model to interpolate the experimental crack growth curves (actual crack length as function of the number of cycles), that is alternative to the methods proposed by the ASTM 647-95a Standard, has been derived analysing the data of 180 fatigue crack propagation tests carried out by Ghonem and Dore. By a cross-check performed by means of the normality test of the residuals and the coefficient of determination $\mathrm{R}^{2}$, it has been highlighted that it is possible to identify those data groups, if present, which move in an anomalous and non influential manner away from the trend of the other data points, so as to be able to take in count of them in the analysis of the results. Furthermore, the model parameters have been evaluated and some interesting correlations have been found between them, which, after further validation by means of an extended experimentation, could be useful to build up a new phenomenological model, that is robust and well-rounded at the same time, to compute the fatigue crack growth rates. 


\section{REFERENCES}

[1] A.K. Head, Phil .Mag., 44(7) (1953) 925.

[2] ASTM E 647, Standard test method for measurement of fatigue crack growth rates, ASTM (1995), Philadelphia, PA, USA.

[3] B. Mukherjee, Int. J. of Fracture, 8(4) (1972) 449.

[4] R. A. Smith, Int. J. of Fracture, 9(3) (1973) 352.

[5] K.B. Davies, C.E. Feddersen, Int. J. of Fracture, 9 (1973)116.

[6] J. Polak, Z. Knesl, Int. J. of Fracture, 11 (1975) 693.

[7] A. De Iorio, D. Ianniello, F. Penta, E. Santoro, In: $8^{\text {th }}$ ECCM, Naples, (1998).

[8] H. Ghonem, S. Dore, Engineering Fracture Mechanics, 27(1) (1987) 1.

[9] S. C. Chapra, R. P. Canale, Numerical Methods for Engineers with PC applications, McGraw-Hill, (1985). 